\title{
CONDITIONS OF STRESS CORROSION CRACK GROWTH AND RETARDATION IN X70 STEEL IN CARBONATE ENVIRONMENTS
}

X70 steel has been often used for building of high pressure transit pipelines. In spite of multiple pipeline protection being used, namely insulation and cathodic protection, in complicated soil and environmental conditions, some pipeline sections can be exposed to corrosive environments. Seriousness of stress corrosion cracking (SCC) damage in carbonate environments is being sometimes flouted by some operators. The work was aimed at qualitative and quantitative analyses of SCC process and damage mechanisms under static loading with individual unloading cycles in standard carbonate solution at room and elevated temperatures. SCC growth was demonstrated as well as an importance of stress fluctuations affecting the process.

\section{Introduction}

A considerable part of Czech gas and oil pipelines have been in service already for many years. In recent years, non-destructive inspections or several high-pressure pipeline crashes indicated a significant amount of stress corrosion cracking (SCC) damage. In this context, research programmes aimed at an investigation of sensitivity of high pressure pipeline steels to SCC and connected damage mechanisms have become important.

Numerous cases of SCC damage to transit pipelines in areas containing alkaline soil and carbonates have been observed not only worldwide. (e.g. [1]), but also in the Czech Republic. An occurrence of carbonates in the soil can be supported by cathodic protection potentials [2]. When an insulation layer is damaged, for instance during pipeline mounting or land up, or in case of cathodic protection dysfunction, SCC damage to pipe external surface can be usually observed [3]. Such a case also occurred in Slovakia near Borsky Mikulas in 1988. After the explosion, the pipe was damaged by $25 \mathrm{~m}$ long spiral crack, in parallel with the weld [3].

SCC process usually has three characteristic phases of sigmoidal shape of the time dependence, (i) relatively fast microcrack initiation followed by (ii) quite lengthy microcracks development and formation of the main crack and evetually (iii) SCC growth of the main crack to failure. Previous works in the field of SCC in alkaline environments performed at SVÚM were focused on investigations of the first stage [4]. This paper contains results of a research programme aimed at an investigation of SCC growth of already existing long cracks and at verification, whether and under which conditions such a process occurs and what its mechanisms are.

\section{Experiments}

Experiments were performed on an X70 steel having been recently often used particularly for building of high-pressure transit pipelines not only in our country, but also in many worldwide regions. Evaluation of reliability and safety of pipelines made of this steel has been recently a matter of numerous extensive research programmes (e.g. [5]).

Specimens were taken from a sheet thermomechanically treated using standard procedures, resulting in a characteristic line type, orientated, ferritic-pearlitic microstructure. Specimens were of a nonstandard CT-type (Fig. 1), with an increased length to enable to attach chambers for the corrosive solution (Fig. 2).

Direct current potential drop (DCPD) method with analytical calibration was used to measure a crack length and growth $[6,7]$. Potential cables were initially connected in the vicinity of a notch (crack) mouth to get the highest possible sensitivity. The cables were therefore inserted into the chamber - Fig. 2. However, during the long-time tests, the cable attachment in the solution was corroded off. Therefore, later on, potential cables were connected outside the chamber, approximately $20 \mathrm{~mm}$ from the notch. This measure resulted in a reduced sensitivity, but made the measurement reliable.

An initial crack was prepared by fatigue pre-cracking in air, at a load frequency of $30 \mathrm{~Hz}$, the asymmetry $R=0.5$, a load range of $9 \mathrm{kN}$ and $7.5 \mathrm{kN}$ in the first and second stages, respectively. The final crack length was approximately $21.5 \mathrm{~mm}$, the stress intensity factor range at the final stage $\Delta K=19.5 \mathrm{MPam}^{1 / 2}$, i.e. $K_{\max }=$ $=39 \mathrm{MPam}^{1 / 2}$.

\footnotetext{
* Ivo Cerny, Dagmar Mikulova, Pavel Novak

SVUM a.s., Praque, Czech Republic, E-mail: Ivo.Cerny@seznam.cz
} 


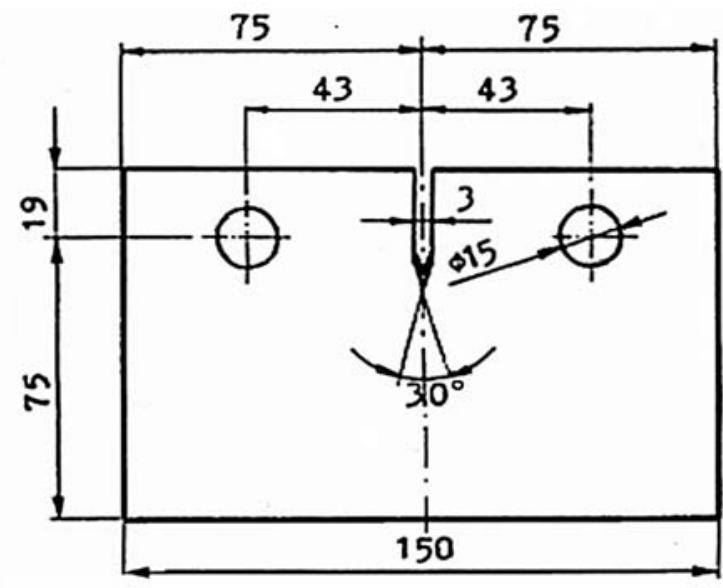

Fig. 1 Non-standard CT specimen used

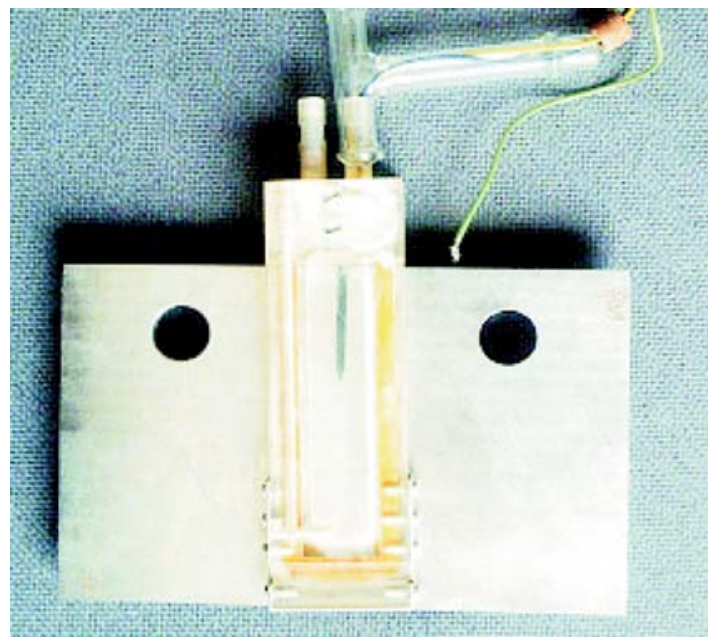

Fig. 2 Actual specimen with chamber for corrosive solution and potential cables

The alkaline corrosive solution simulating real carbonate environments was prepared according to [8], namely mixture of 1normal sodium carbonate and 1-normal sodium bicarbonate solutions in the ratio of 1:1. Resulting $\mathrm{pH}$ was 9.8. SCC experiments were performed at a static load of $36.6 \mathrm{kN}$, with the resulting $K=$ $=90 \mathrm{MPam}^{1 / 2}$, that means at rather high loading. The corrosive medium was circulating through the chamber. Three specimens were tested: (i) specimen designated $\mathrm{X} 75$ at an elevated temperature of $70{ }^{\circ} \mathrm{C}$, (ii) $\mathrm{X} 76$ at room temperature and (iii) X78 at room temperature with a long-time exposure. The elevated temperature had practical reasons: Gas is usually considerably warmed-up when passing through compression stations in transit pipelines.

Due to possible fracture surface passivation in alkaline environments, unloading cycles corresponding to the load asymmetry $R=0.1$ started to be applied after some initial stage of the experiments. Unloading cycles also are linked to real conditions, they correspond to pressure fluctuations or pipeline service interruptions.
All the tests were finished after interruption of the static loading stage with individual unloading cycles, with fatigue loading at the same load range $\left(F_{\max }=36.6 \mathrm{kN}, R=0.1\right)$, with reduced load frequency $f=0.05 \mathrm{~Hz}$, without interrupting the circulation of the corrosive solution. Eventually, the test programme was completed with a reference test of specimen X77, where the same loading sequence was applied including the final fatigue stage, but without any corrosive environment.

Fracture surfaces, particularly zones following fatigue pre-cracking, where SCC mechanisms were expected, were fractographically analysed using a scanning electron microscope JEOL JSM 35.

\section{Experimental results and discussion}

\subsection{Theoretical DCPD calibration}

Analytical calibration was calculated for all the configurations of potential electrodes used. It should be mentioned that potential was measured not only near the crack mouth $(V(a))$, but also a reference potential $V_{\text {ref }}$ was recorded to compensate temperature changes and the ratio $V(a) / V_{\text {ref }}$ was evaluated $[6,7$,$] . The results$ are shown in Fig. 3. s

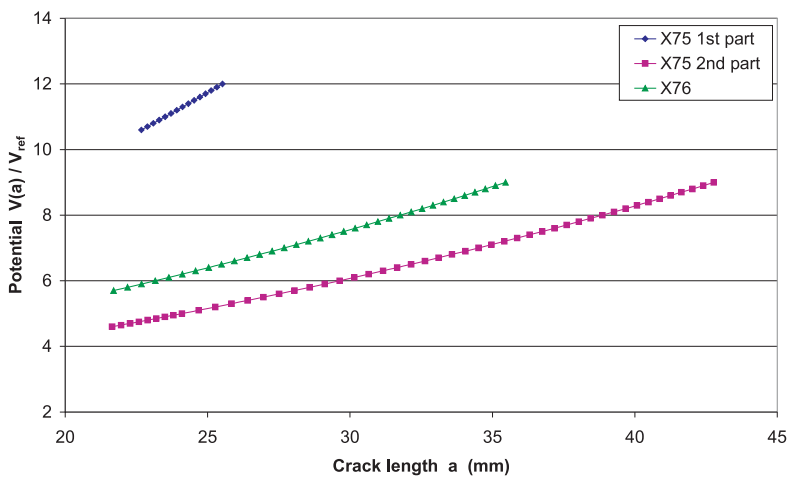

Fig. 3 Analytical calibration curves of the DCPD method used

\subsection{Stress corrosion crack growth}

Results of DCPD measurement during the SCC test of the specimen X75 tested at $70{ }^{\circ} \mathrm{C}$ are shown in Fig. 4. The points correspond to individual measurement sequences and represent average values of more than 10 potential measurements. There are two stages in the diagram, which shows as a whole a continuous, not interrupted test. The first stage, initial increase of the potential corresponding to 1 hour exposure, can be an artefact connected or at least affected by temperature balancing. This stage corresponds to the temperature growth of the solution from room temperature to $70{ }^{\circ} \mathrm{C}$. The next 8 hours stage, however, already indicates some initial SCC growth with a saturation effect at the end. Further exposure at these conditions and temperature did not lead to further significant increase of potentials. The total potential increase 
$V(a) / V_{\text {ref }}$ at the second stage 0.25 is not negligible, it corresponds approximately to more than $0.5 \mathrm{~mm}$ crack increment.

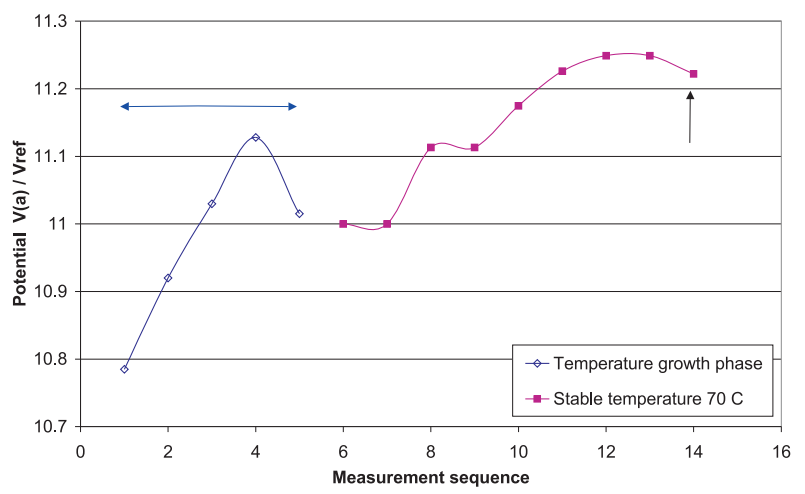

Fig. 4 Potential measurement during SCC test of specimen X75 at $70^{\circ} \mathrm{C}$

The results indicate that the characteristic first stage of the SCC process was monitored during the 8-10 hours measurement, namely quite fast crack growth, which is typically followed by a retardation of the process and long-time development phase. The retardation may also be affected by an occurrence of corrosion products near the crack tip resulting in a restricted access of the corrosive medium to the crack front.

The second experiment, carried out on another specimen of the X70 steel designated X76, was performed at room temperature. Details of the DCPD measurement at two important phases, namely after starting the corrosive solution input into the chamber and after starting individual unloading cycles, respectively, are shown in Figs. 5 and 6. In the diagrams, individual recorded points connected with a considerable scatter are plotted together with moving average, better indicating changes of the time dependence.

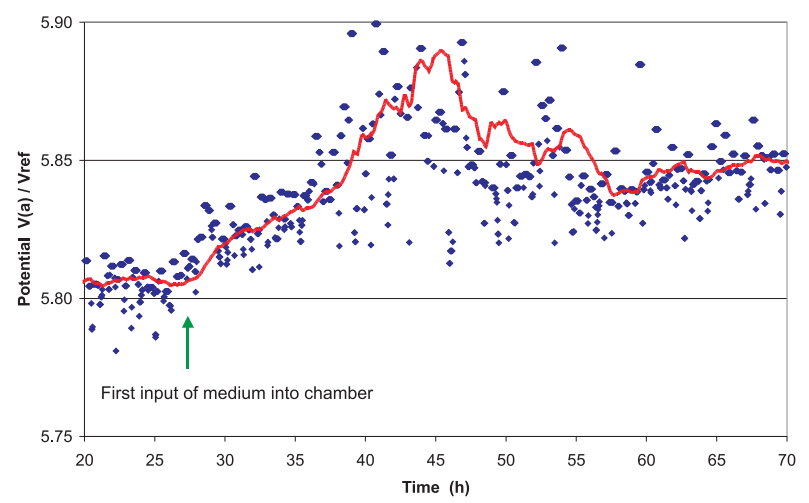

Fig. 5 Potential measurement during SCC test of specimen X76 at room temperature, the phase following the first medium input into chamber

Before the first input of the medium into the chamber, average DCPD values were constant, as expected. However, a distinct increase of the potential values can be seen next 17-18 hours, fol-

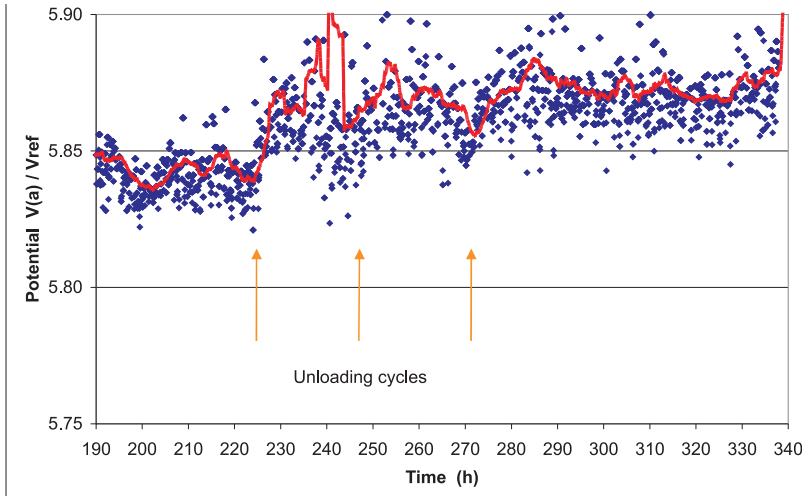

Fig. 6 Potential measurement of specimen X76 at room temperature, the phase with unloading cycles

lowed then by saturation and even a slight decrease. It is the same tendency like in the previous test at $70{ }^{\circ} \mathrm{C}$ with the difference that at room temperature, the process was approximately two times longer. Almost no changes were then observed until 225 hours of the exposure, when three unloading cycles were gradually applied with the interval of 24 hours. In spite of the scatter, the moving average of potentials shows potential increase after each of the unloading cycle followed by fairly small decrease, the most intensive increase occurring after the first of the three cycles. This effect may be connected with microcracking of a passivation layer being most important after the first cycle, which occurred after a long period with purely constant static load. The effect of the second and third unloading cycles, when the passivation likely was not so strong due to the short time of constant load, just 24 hours, seems not to be so strong. However, the total increase after the three cycles is evident.

The last SCC experiment, specimen designated X78, was carried out exactly at the same conditions as the previous one, i.e. at room temperature with the only differences that more than three unloading cycles were applied and the whole experiment was performed for a longer time, almost three times. Unloading cycles were applied manually during weekdays in the morning and late afternoon, starting after 160 hours of exposure in the solution just with a constant static load. Unloading was, however, inter-

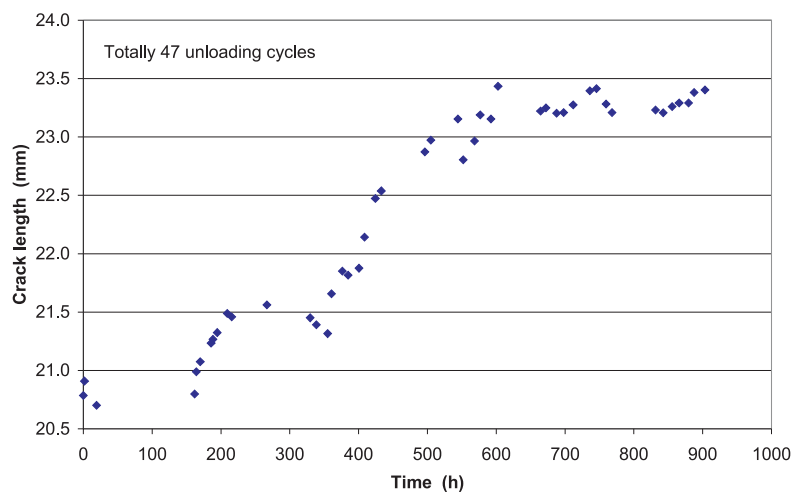

Fig. 7 SCC growth in specimen X78 at room temperature 
rupted for the 115 hours period, between 215 and 330 hours of the exposure.

Results are shown in Fig. 7 Each point represents the average value of 20 measurements. In this diagram, the potentials are already recalculated to the actual crack length. This experiment confirmed without doubt what was already indicated during the previous one: an importance of unloading cycles in the SCC growth process at carbonate solution in the $\mathrm{X} 70$ steel. On the contrary, when load fluctuation occurred, SCC growth was quite rapid. The results confirm seriousness of SCC growth in carbonate environments in actually operated pipelines, unlike numerous allegations of particularly domestic operators that such the process cannot occur.

Some minor remarks concern two phases of the experiment: (i) the beginning, when the initial crack growth was observed as before (not distinctly recorded in the diagram in Fig. 7), followed by the saturation to 160 hours and (ii) a distinct saturation of the potentials between 215 and 330 hours, when application of unloading cycles was temporarily discontinued. The potential decrease may be caused by increasing contact of fracture surfaces near crack tip due to growth of corrosion products.

\subsection{Fractographical analysis}

Fracture surfaces of specimens X75 (tested at $70{ }^{\circ} \mathrm{C}$ ), X76 (tested at room temperature) and X77 (loaded just in laboratory air without corrosive solution) were analysed, with a special attention to the zones of crack growth supported by SCC mechanisms, i.e. in the zones between the end of fatigue precracking and following approximately $0.3-1 \mathrm{~mm}$ crack increments.

There are some characteristic features common for both the specimens tested in the corrosive solutions, proving existence of SCC mechanisms: an occurrence of numerous secondary cracks confirming strong branching mechanism typical for SCC processes.

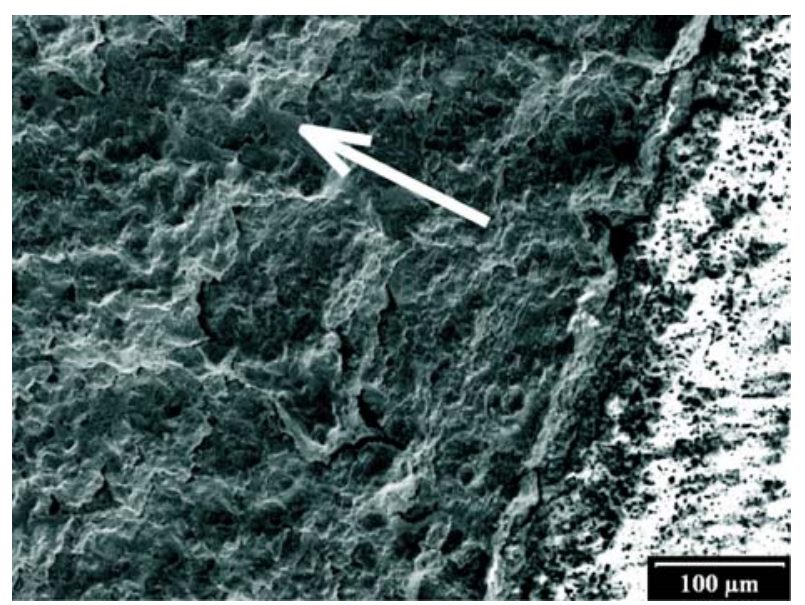

Fig. 8 Example of secondary cracks in region of SCC growth in specimen $X 75$
Fig. 8 represents an example of such secondary cracks in specimen X75. Crack growth direction is indicated by the arrow. The white part of the Figure in the right margin represents the fracture surface of fatigue precracking, strongly corroded during the next exposure in the warm carbonate solution. Numerous deep secondary stress corrosion (SC) cracks can be seen in the boundary line between fatigue precracking and SCC growth. These cracks are mostly parallel to the main crack front. At the distance up to $0.3 \mathrm{~mm}$ from the boundary line, further secondary SC cracks, more branched and with more complicated directions can be observed.

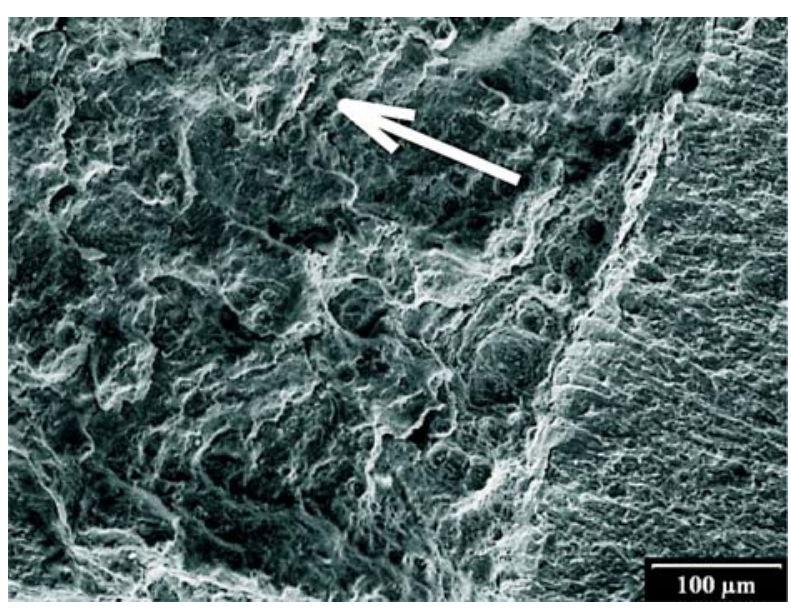

Fig. 9 Secondary cracks in region of SCC growth in specimen X76 (room temperature)

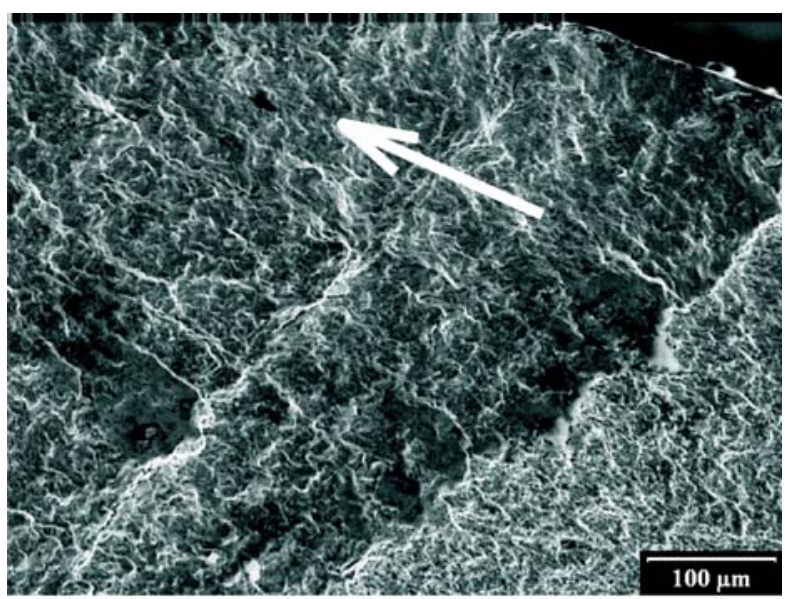

Fig. 10 Fracture surface without secondary SCC cracks in specimen $X 77$ loaded in air

In spite of some common features, fracture surface of specimen X76 tested at room temperature is rather different from X75 - Fig. 9. First of all, no huge corrosion products occurred on the fatigue precracked surface, indicating that surface corrosion at room temperature was suppressed. On the other hand, secondary SC cracks could be observed in a similar way as in Fig. 8 - a zone of secondary cracks parallel to the boundary line and numerous $\mathrm{SC}$ cracks with a complicated shape in the region, where crack 
growth was supported by SCC mechanisms. On the contrary, the fracture surface of specimen X77, loaded with the same sequence as the previous two specimens, was completely different - Fig. 10 . The secondary cracking characteristic for the stress corrosion process could not be observed in this case.

\section{Conclusions}

The experimental programme was focused on qualitative and quantitative analyses of stress corrosion crack process and damage mechanisms in an X70 steel under static loading with individual unloading cycles, exposed to carbonate solution, at room and elevated $\left(70{ }^{\circ} \mathrm{C}\right)$ temperatures. The most important results can be summarised as follows:

- The results proved without doubt a significant occurrence of SCC growth in the X70 steel in carbonate environments. This result contrasts with numerous allegations of some pipeline operators that there is no danger of SCC process at these conditions.
- An importance of unloading cycles in the SCC growth process in carbonate solution was shown. When the loading was purely static without fluctuations, damaging the passivation layer near the crack tip, SCC growth was saturated after some time and even some decrease of measured potentials could be observed. On the contrary, when load fluctuations were applied, SCC growth was quite rapid. SCC growth seemed to be somewhat more progressive at elevated temperature.

- Fractographical analyses proved an existence of SCC growth mechanisms, showing numerous secondary strongly branched cracks on fracture surface in the region of SCC growth. Such cracks were observed on fracture surfaces of specimens tested in solutions at both room and elevated temperatures.

\section{Acknowledgement}

The work was carried out within the project supported by the Czech Ministry of Education, Youth and Sport under grant MSM 2579700001.

\section{References}

[1] PARKINS R. N.: Stress Corrosion Cracking of Pipelines - Its Control or Prevention Corrosion, Paper No. 249, NACE International, 1996.

[2] LEIS, B.-N., PARKINS, R.-N.: Mechanics and Materials Aspects in Predicting Serviceability Limited by SCC. Fatigue \& Fracture of Eng. Mater. V. 21, 1998, p. 583-601.

[3] LINHART V., SIGMUNDOVA J., RABL V.: Resistance of Steels Applied in Gas Transmission Pipelines to Stress Corrosion Cracking. Slovgas, Vol. 5, 2008, pp. 12-15 [in Czech].

[4] CERNY I., LINHART V.: An Evaluation of Resistance of Pipeline Steels to Initiation and Early Growth of Stress Corrosion Cracks. Engng. Fracture Mech., Vol. 71, 2004, pp. 913-921.

[5] SHANGHAI RESEARCH INSTITUTE OF MATERIALS (SRIM): Research on Stress Corrosion Cracking of Engineering Materials. Power Point presentation, ME08118 project workshop, SVUM Praha, 2008.

[6] CERNY, I.: The Use of DCPD Method for Measurement of Growth of Cracks in Large Components at Normal and Elevated Temperatures. Engng. Fracture Mech., Vol. 71, 2004, pp. 837-848.

[7] CERNY, I.: Measurement of Subcritical Growth of Defects in Large Components of Nuclear Power Plants at Elevated Temperatures. Int. J. of Pressure Vessels and Piping, Vol. 78, 11/2001, pp. 893-902.

[8] WANG, Z. F., ATRENS A.: Initiation of Stress Corrosion Cracking for Pipeline Steels in a Carbonate-Bicarbonate Solution, Metallurgical and Materials Transactions, Vol. 27A, 1996 